\title{
Co-Creation of HIVST Delivery Approaches for Improving Urban Men's Engagement with HIV Services in eThekwini District, KwaZulu-Natal: Nominal Group Technique
}

Tivani Mashamba-Thompson

University of Pretoria

Richard Lessells

Duke Medical Research Institute

Tafadzwa Dzinamarira ( $\sim$ anthonydzina@gmail.com )

University of Pretoria School of Health Systems and Public Health https://orcid.org/0000-0002-99295739

Paul Drain

University of Washington Seattle Campus: University of Washington

Lehana Thabane

McMaster University

\section{Research Article}

Keywords: Men, Urban, HIV self-testing, Delivery

Posted Date: August 30th, 2021

DOI: https://doi.org/10.21203/rs.3.rs-805091/v1

License: (c) (i) This work is licensed under a Creative Commons Attribution 4.0 International License.

Read Full License 


\section{Abstract}

Background: HIV self-testing (HIVST) is one of the recommended approaches for HIV testing services, particularly for helping reach populations who would not normally access facility-based HIV testing. HIVST must be tailored to different populations to ensure uptake.

Objective: The main objective of this study was to develop an acceptable HIVST delivery strategy to help improve urban men's engagement with HIV services.

Methods: We invited key stakeholders for urban men's HIV services to participate in a co-creation workshop aimed at developing HIVST delivery approaches for urban men, using eThekwini municipality as a study setting. We conducted purposive sampling to include health care users and health care providers, representing a range of views across the public sector and voluntary sector. We employed the Nominal Group Technique (NGT) method for data collection. The NGT workshop was conducted in two consecutive phases: phase one was focused on determining barriers for men's engagement with the current/facility-based HIV testing services; phase two was aimed at determining HIVST delivery strategies. We used the results of the NGT to design a tailored HIVST strategy for urban men in eThekwini District.

Results: Participants identified the following psychological factors as the most important barriers to uptake of HIV testing services by urban men: stigma, ignorance about the importance of testing and testing process as well as fear of positive test results. Key stakeholders suggested internal motivation strategies as a potentially effective approach to support HIVST delivery strategy. Guided by the NGT results, we designed a HIVST delivery strategy that is supported by a risk communication approach.

Conclusion: We designed an evidence-based risk communication mobile health (mHealth) strategy coupled with SARS COV-2 self-testing tailored to improve men's uptake of HIVST. A follow-up study to evaluate the feasibility of implementing these approaches is recommended.

\section{Key Messages}

1) Across sub-Saharan Africa, men living with HIV are $20 \%$ less likely than women living with HIV to know their HIV status. HIV self-testing (HIVST) presents an opportunity to improve testing rates among this underserved population, however tailored delivery approaches are required.

2) This paper describes the design of an intervention, a HIVST delivery strategy that is supported by a risk communication approach

3) Improving the uptake of HIV testing by developing tailored HIVST delivery strategies for underserved population such as urban men is key to reducing new HIV infections

\section{Background}


Across sub-Saharan Africa, men living with HIV are $20 \%$ less likely than women living with HIV to know their HIV status $(1,2)$. This presents a major public health problem as knowledge of one's status is the first and most important step in the HIV care and treatment cascade (3). Approximately, 7700000 people were living with HIV in South Africa and the rate of new infection remains high (4). South Africa's HIV programme is the largest in the world and has been making progress towards achieving the Joint United Nations Programme on HIV/AIDS 90-90-90 targets ( $90 \%$ living with HIV know their status, $90 \%$ of these on antiretroviral treatment, and $90 \%$ with undetectable viral loads) (5). However, most of the gains in achieving the targets have occurred in females living with HIV, while gains in males living with HIV have been modest (5). There are substantial gaps in HIV service use and coverage for men and boys in South Africa (6). There is evidence to suggest that, overall, men have lower levels of engagement and retention in HIV care $(7,8)$ and higher mortality on ART than women (9). The observed differences in mortalities may be best explained by background differences in mortality between men and women may be related to other factors including men's poor usage of facility-based health services.

By 2020, at least 400,000 more men in South Africa need to take regular HIV tests and commence treatment to ensure that the country achieves its target of providing treatment to $90 \%$ of all men and women testing positive (10). To reach the 400,000 men in South Africa efforts to create HIV testing services that are tailored to meet men's needs are urgently needed. Community-based HIV testing had a significant effect on reaching a high number of HIV-positive men when compared to facility-based testing (11). HIVST is one of the WHO recommended approaches for HIV testing services (12), particularly for helping reach populations who would not normally access facility-based HIV testing. For larger-scale dissemination of community-based interventions such as HIVST (HIVST) to be effective, there is a need to understand the processes required to implement the intervention consistently and at a high level of quality, especially implementing the intervention in different contexts (13). HIV self-tests are available to the public in South Africa and they can be purchased via pharmacy outlets. HIVST provides a novel and currently severely underutilized supplement to facility-based testing (14). Oral HIVST offers the potential for increased HIV testing uptake and greater convenience and privacy as well as the potential to increase the proportion of the population who test regularly (14). Evidence on the acceptability of HIVST in subSaharan Africa has suggested higher acceptability of HIVST among men than women (15).

In general, self-testing offers people a self-management solution - empowers people to be in control of their care. In terms of HIV, a highly stigmatised condition, there are many effective ways to deliver and support HIVST (HIVST), depending on the population and setting (16). Most HIV-related research on marginalised populations prioritises rural populations as they are often conducted in rural settings. This poses a risk to a delay in addressing the needs of marginalised urban settings. Urbanization is one of the fast-growing global trends of the 21st century. Approximately, 2.5 billion more people will be added to the urban population by 2050, mainly in Africa and Asia (17). Urbanisation can result in a significant on individual quality of life, while straining public health systems and resources (17)(18). There is limited evidence on the most appropriate delivery strategies for HIVST to enable urban men to engage with HIV services in South Africa. HIVST has recently been introduced as a supplementary HIV testing strategy in South Africa and recommended to reach the key and under-tested populations (19). Therefore, it is 
important to use appropriate delivery strategies to maximize uptake and impact. It is also recommended that relevant stakeholders are involved in developing and adapting HIVST models. The main objective of this study was to collaborate with stakeholders in the development of HIVST delivery approaches to help improve urban men's engagement with HIV services. It is anticipated that the results of this study will help guide a planned intervention study to determine the most acceptable delivery strategy for urban men.

\section{Methods}

We used the Nominal Group Technique method for this study. We invited key stakeholders for urban men's HIV services such as health care providers, health care service users, government employees, NGOs and academics to participate in a co-creation workshop aimed at developing HIVST delivery approaches for urban men, using eThekwini municipality as a study setting. We purposely sampled our participants. We defined stakeholders as people who have expert knowledge on HIV services, men's health services and have an interest in the implementation of HIVST for men in KwaZulu-Natal, South Africa. During the workshop, we employed the Nominal Group Technique (NGT) method for data collection (20). The NGT enabled problem identification, solution generation, and decision making among stakeholders. We conducted the workshop in two consecutive phases: phase one was focused on determining barriers for men's engagement with the current/facility-based HIV testing services; phase two was aimed at determining HIVST delivery strategies to help improve urban men's engagement with HIV services.

\section{Phase one}

We requested key stakeholders to share their knowledge on barriers to urban men's engagement with current (facility-based) HIV testing services. Following instructions from the facilitator, stakeholders independently grouped their suggestions into themes. The PI (TPM-T) listed the themes in a voting form/questionnaire to enable voting through ranking. Participants were requested to rank the themes according to the level of severity in preventing men's engagement with current (facility-based) HIV testing and treatment services. The ranking score was between one and five.

\section{Phase two}

Key stakeholders were requested to propose potential HIVST delivery strategies to help improve urban men's engagement with HIV-self-testing and to group them according to themes, without the PI's assistance. The PI listed the themes in a voting form/questionnaire to enable voting through ranking. Participants were requested to rank the themes according to the level of effectiveness to enable the delivery of HIV-self testing to urban men. The ranking score was between one and five, one being least effective and five being most effective strategy.

Following the NGT workshop, a report presenting the results of NGT was put together by the TPMT and shared with key stakeholders for comments.

\section{Data Management and Analysis}


To obtain the quantitative data gathered during the ranking step in the nominal group process, a total importance score for each barrier was calculated by summing the individual scores of the participants. The ranking scores were between one and five, one being the least severe, and five being the most severe barrier to urban men accessing HIV self-testing. The qualitative data were analyzed using thematic content analysis to inductively identify the themes that emerged from the data presented during the discussion. The coding categories were derived directly from the text data to limit researcher biases due to preconceived ideas or other theoretical perspectives.

\section{Results}

Eleven HIV key stakeholders aged 18-60 representing different population groups agreed to participate in our workshop. Of these, $73 \%$ were male. The majority (64\%) of the study participants were employed, two were unemployed and two were full-time students. Characteristics of participants are presented in Table 1 below:

Table 1

Characteristics of workshop participants

\begin{tabular}{|lll|}
\hline Gender & $\begin{array}{l}\text { Age group } \\
\text { (years) }\end{array}$ & Occupation \\
\hline Male & $56-65$ & Unemployed \\
\hline Male & $26-40$ & Health Data specialist \\
\hline Male & $26-40$ & High School Teacher \\
\hline Female & $41-55$ & Postdoctoral Student- researching patient-centeredness of HIV services \\
\hline Male & $26-40$ & Lecturer \\
\hline Male & $18-25$ & Full-time Undergraduate Student \\
\hline Male & $41-55$ & Research Project Director for an international NGO \\
\hline Male & $41-55$ & HIV services Program Manager \\
\hline Female & $26-40$ & HIV counsellor \\
\hline Male & $56-65$ & $\begin{array}{l}\text { Former Piping Supervisor, current volunteer HIV mentor and counsellor } \\
\text { construction industry employees }\end{array}$ \\
\hline Male & $18-25$ & Full-time Masters Student \\
\hline
\end{tabular}

Stakeholders reported thirteen factors as urban men's barriers to accessing facility-based HIV testing services (Fig. 2). The voting results showed that stigma as the most highly ranked barrier (55 scores) followed by ignorance ( 50 scores), fear, (49 scores) lack of knowledge on the use of HIVST kits (46 scores) and psychology (43 scores), respectively. Lack of incentives (34 scores) was voted as the least severe barrier followed by affordability ( 35 scores) and health services (38 scores), respectively. 
Table 2

Suggested HIVST priority strategies

\section{Priority Key stakeholder's suggestions strategy}

Internal motivation to help encourage

"It's can be applied to men to engage in an activity because they like it, make them feel good and helps them to take ownership of their personal life/health. The Department of health may engage to motivate men via workshops, televisions ads and writings on papers (e.g: flyers), school visits may be important too since men engage in physical contacts at young age. Internal motivation may be delivered to men in a form of a story (a person who live with a virus may be a good to share experiences), praises, awards/benefits and recognition."

"The health professionals that do HIV testing should be males.

Males should be given an option to choose the gender they pre-order to test them.

Empathy should be emphasized by the health workers, e.g. I understand your fear, I've also tested etc."

"To adequately deal with the male's self-esteem/fear related issues prior to him engaging in the self-testing process. This requires therapy/counselling with service providers who are passionate about male sexualities/masculinities/sexual reproductive health. Additionally the videos or information packs should be personcentred and speak to various levels of emotional intelligences, backgrounds, etc."

Use of videos for HIVST pre and post testing counselling
"Advocate that HIV self-testing is $100 \%$ private and the outcomes of the results are only seen by the self-tester. The self-testing kit should come in a package that has pens, bottles or t-shirts written "I have tested have you??"

"Through YouTube videos, comprehensively holistic information packs, etc"

"Cinema ads before the movie stars, urban are engaged in social medias such as face book, WhatsApp and tweeter, self-testing videos maybe posted there by the Department of Health and/or other health organisations."

"Pre and post counselling videos should be available on YouTube as it is the biggest video platform. The videos should also be available on the department of health website. The self-testing kit should have a link where the videos are available."

"Data or WIFI opportunities must be available to the person who is considering the selftesting. Videos should be graded incorporating. age, religion, culture, background." 


\begin{tabular}{|c|c|}
\hline $\begin{array}{l}\text { Priority } \\
\text { strategy }\end{array}$ & Key stakeholder's suggestions \\
\hline \multirow{5}{*}{$\begin{array}{l}\text { Promotion } \\
\text { of self- } \\
\text { testing via } \\
\text { social } \\
\text { media }\end{array}$} & "Internet forum, group chats, group page, etc" \\
\hline & $\begin{array}{l}\text { "Through men's health WhatsApp business account. Facebook pages, Twitter } \\
\text { accounts that are created for self-testing. Each community should create a WhatsApp } \\
\text { group that is specifically focused on HIVST that and encourages men to test." }\end{array}$ \\
\hline & $\begin{array}{l}\text { "Community mobilisers, mentor self-testers. Video clips of celebrities promoting self- } \\
\text { testing." }\end{array}$ \\
\hline & $\begin{array}{l}\text { "Male circumcision adverts should include the promotion of self-testing. } \\
\text { advertisements should be focused and based on "self -testing is } 100 \% \text { private and } \\
\text { confidential" as well as emphasizing where they are available be it public health } \\
\text { facilities to stores. As males we tend to see going to the hospital/clinics as something } \\
\text { taboo, therefore it will be much more convenient if self-testing kits are not only } \\
\text { available in hospitals and clinics." }\end{array}$ \\
\hline & $\begin{array}{l}\text { "Use men from various background so individuals feel they are represented and can } \\
\text { identify with the person/s in the adverts." }\end{array}$ \\
\hline
\end{tabular}

A large proportion (73\%) of the participants supported scaling up HIVST to help improve urban men's engagement with HIV testing and treatment services. All eleven participating stakeholders were requested to suggest HIVST delivery strategies and rank them according to their potential effectiveness to help address the above challenges and improve urban men's engagement with HIV services. Figure 3 shows 18 suggested HIVST strategies in ascending order of their ranking score. Key stakeholders ranked the promotion of HIVST via TV adverts as the most desirable (85\%) strategy to help enable uptake HIV-self testing and enable urban men's engagement with HIV testing and treatment services, followed by the use of videos for HIVST pre- and post-testing counselling ( $80 \%)$, promotion of self-testing via social media $(80 \%)$, placing test kits in pubs (80\%), Internal motivation strategies (80\%) and Provision of free testing kits $(80 \%)$. 
Table 3

HIV testing delivery strategies to improve urban men's engagement with HIV testing services

$\begin{array}{lll}\text { Priority delivery strategies for HIVST } & \begin{array}{l}\text { Summing by votes } \\ 1=\text { less effective }\end{array} & \begin{array}{l}\text { Total number of voting scores } \\ \text { (weighted sum }=\text { number of votes } \times \\ \text { ranking score) }\end{array} \\ & 5=\text { highly effective }\end{array}$

$\begin{array}{lllllll}1 & 2 & 3 & 4 & 5 & 55\end{array}$

$\begin{array}{lllllll}\text { Using celebrities to promote uptake of } & 1 & 1 & 3 & 5 & 1 & 37\end{array}$ HIVST

$\begin{array}{lllllll}\text { Using endorsement } \text { of HIV self-tests by } & 1 & 1 & 4 & 2 & 3 & 38\end{array}$

Department of Health

$\begin{array}{llllll}\text { Using sports figures to promote uptake } & 2 & 3 & 4 & 2 & 39\end{array}$ of HIVST

Providing incentives

$\begin{array}{lllll}2 & 4 & 1 & 4 & 40\end{array}$

$\begin{array}{llllll}\text { Incorporating mHealth within HIV self- } & 1 & 4 & 3 & 3 & 41\end{array}$

testing

$\begin{array}{lllllll}\text { Partner testing } & 1 & & 4 & 1 & 5 & 42 \\ \begin{array}{l}\text { Giving men a reason to believe in HIV } \\ \text { testing }\end{array} & 1 & 4 & 2 & 4 & 42 \\ \begin{array}{l}\text { Packaging HIVST kits with STI } \\ \text { (sexually transmitted disease) self- } \\ \text { tests }\end{array} & 1 & 3 & 4 & 3 & 42 \\ \end{array}$

$\begin{array}{llllll}\text { Packaging HIV self-tests kits with other } & 2 & 2 & 3 & 4 & 42\end{array}$ diseases screening self-tests

$\begin{array}{llllll}\text { Providing clear instructions } & 1 & 2 & 5 & 3 & 43\end{array}$

$\begin{array}{llllll}\text { Placing test kits in community centres } & 1 & 1 & 5 & 4 & 43\end{array}$

$\begin{array}{llllll}\text { Provision of free data } & 1 & 1 & 6 & 3 & 43\end{array}$

$\begin{array}{llllll}\text { Provision of free testing kits } & 1 & 2 & 3 & 5 & 44\end{array}$

$\begin{array}{lllll}\text { Internal motivation strategies } & 3 & 5 & 3 & 44\end{array}$

$\begin{array}{llllll}\text { Placing test kits in pubs } & 1 & 1 & 4 & 5 & 44\end{array}$

$\begin{array}{lllllll}\begin{array}{l}\text { Promotion of self-testing via social } \\ \text { media }\end{array} & 1 & 1 & 5 & 4 & 44\end{array}$

$\begin{array}{llllll}\text { Use of videos for HIVST pre and -post- } & 1 & 7 & 3 & 44\end{array}$
testing counselling

Please define all acronyms used in the table below it 


\begin{tabular}{|c|c|c|c|c|c|c|}
\hline \multirow[t]{2}{*}{ Priority delivery strategies for HIVST } & \multicolumn{5}{|c|}{$\begin{array}{l}\text { Summing by votes } \\
1=\text { less effective } \\
5=\text { highly effective }\end{array}$} & \multirow{2}{*}{$\begin{array}{l}\text { Total number of voting scores } \\
\text { (weighted sum }=\text { number of votes } \times \\
\text { ranking score) } \\
\\
55\end{array}$} \\
\hline & 1 & 2 & 3 & 4 & 5 & \\
\hline $\begin{array}{l}\text { Promotion of self-testing via TV } \\
\text { adverts }\end{array}$ & 1 & & & 4 & 6 & 47 \\
\hline \multicolumn{7}{|c|}{ Please define all acronyms used in the table below it } \\
\hline
\end{tabular}

\section{Reported barrier versus proposed strategies}

Our results show a relationship between the most important barriers to accessing HIV testing and some of the suggested HIVST strategies by stakeholders. The use of internal motivation strategies has been proposed as a strategy that could help address three of the most important barriers to accessing HIVST by urban men - stigma fear and psychology (Table 4).

Table 4

Matching the urban men's barriers to accessing HIV testing services with proposed HIV self-testing delivery strategies

Urban men's barriers to accessing HIV HIVST delivery strategies
services

Stigma

Lack of knowledge on the use of HIVST

Ignorance
Internal motivation strategies

Use of videos for HIVST pre- and post-testing counselling

Promotion of self-testing via TV adverts

Promotion of self-testing via social media

Fear

Psychology
Internal motivation strategies

Internal motivation strategies

\section{Please define all acronyms used in the table below it Concerns relating to scaling up of HIVST}

The majority of participating key stakeholders supported the scaling up of HIV self-testing to underserved populations. It is worth noting that some of the stakeholders did not support the scaling up of HIVST to these populations and they raised some concerns. These stakeholders were concerned about HIVST data availability, adequacy of pre and post-testing counselling and patients' reactions to positive test results. 
'The major issue is that usage of the HIV test kits as we cannot have proof of the results. All depend on the client to say whether or not they have used it. No data on how many negative or positive clients use tested and how many confirmed test results. It is hard to get this information, except to say how many self-screening kits were distributed. This is similar to condoms, as we see the number of sexually transmitted diseases increase while the number of condom distribution increases.'

\section{HIV Counsellor}

'We can broadcast a lot of places and strategies for men to access HIVST but what happens when a person is tested without proper counselling, I think we should introduce a lot of information before scaling up HIVST.'

\section{HIV mentor and counsellor construction industry employees}

'I don't believe that self-testing will work because we are going to experience more suicides. There will also be an issue with anger, if he finds out on his own and this could lead to more cases of rape and abuse. This will be the start of a fire that will not be stopped. It's dangerous, I am speaking from a point of view as I am 12 years diagnosed HIV positive and I deal with HIV positive men and women on a daily bases.'

\section{Feedback from stakeholders and suggestions of priority delivery strategies}

All 11 workshop participants were requested to comment on the proposed approaches to delivering HIVST to urban men. Of these, all read the report and four provided feedback to the report and provided additional suggestions on the implementation of the suggested priority delivery strategies: internal motivation to help encourage; use of videos for HIVST pre and -post-testing counselling; promotion of self-testing via social media; suggested delivery strategies for HIVST for urban men.

\section{Please define all acronyms used in the table below it}

Additional suggestions were provided as part of the feedback to the workshop report to help ensure implementation HIVST to urban men.

Stickers promoting self-testing may be pasted on cars, taxis and trains. Self/ testing kits should available in local stores such as clicks, medirite, pharmacies, Shoprite, spar etc.

Kits should not be expensive so that even unemployed males, pupils and students can access them. Selftesting kits should also be available in universities and schools.

\section{Discussion}


A collaboration with key stakeholders has enabled collective identification of the most important barriers to accessing current HIV testing and treatment services by urban men and identification of priority areas to be considered while developing HIVST delivery approaches for urban men. It has also revealed that psychological factors as the most important barriers to accessing HIV testing and treatment services by urban men. These included stigma, ignorance, fear and lack of knowledge. The World Health Organization refers to poor uptake of HIV services by key populations such as men as a "blind spot" in the global HIV response (1). Key stakeholders suggested the following priority areas be considered during the development of delivery approached for HIVST for urban men: promotion of self-testing via TV adverts; use of videos for HIVST pre- and post-testing counselling; placing test kits in pubs; promotion of self-testing via social media; provision of free testing kits; internal motivation strategies, respectively.

The benefits of promoting health interventions using television as a means to ensure mass communication and raise awareness has been well established in other studies. In Botswana and Zambia television adverts improved uptake of condoms $(21,22)$. Television adverts also improved uptake of voluntary counseling and testing in Ethiopia (23). Key stakeholders also underscored the need for videos for pre- and post- test counselling. HIVST implementation with online video pre- and post- test counselling improved HIV testing coverage and repeated HIV testing among Chinese MSM (24). In Malawi, Zambia and Zimbabwe placing HIVST kits pubs and beerhalls improved uptake among men. Similar recommendations were made by key stakeholders in our study (25).

SSA as a region remains the fastest growing market for smartphone and internet connectivity. $39 \%$ of Africans use mobile internet with an anticipated 10\% growth by 2025 (26). Key stakeholders recommended the promotion of HIVST via social media platforms. This aligns with the findings of a systematic review and meta-analysis that showed social media platforms to improve uptake of HIV services among key populations in LMICs (27). Given the rapid increase in smartphone access and internet connectivity, social media platforms could be instrumental in improving awareness and ultimately uptake. The provision of free testing kits also emerged as an important component of HIVST delivery strategies that improve uptake. This finding corroborates findings from earlier studies conducted among key stakeholders and health care workers in South Africa $(28,29)$. While free provision of test kits it admirable, and likely to result in more coverage, the financial feasibility to support scale-up should also be considered. Ky stakeholders in the current study advocated for internal motivation strategies to improve uptake of HIVST. Internal motivation strategies that improve men have been recommended for South African men in an earlier study (30).

The collaboration with stakeholders on co-creation of delivery strategies for HIVST for urban men resulted broad range of scenarios around how to support the delivery of HIVST for urban men in KwaZulu-Natal South Africa and other similar settings. Based on the demographics of the stakeholders that participated in this study, we recognise that urban men are not a homogeneous group and that urban environments also have disparities in socioeconomic status. Therefore, in order to eliciting HIVST delivery preferences for different groups of men in preparation for implementation, we propose to conduct a discrete choice experiment (DCE). Bearing in mind the current pandemic and the stigma associated with

Page $11 / 16$ 
HIV testing, we recommend the incorporation of SARS COV-2 self-testing to help improve the uptake of HIVST strategies for urban men.

\section{Strengths and limitations of the study}

This study included key stakeholders from different populations groups to collaborate in the cocreation of HIVST delivery approach for urban men. As a result, views different groups of urban men were represented in the creation of these approached. Inclusion different key stake holders enabled us to generate a broader range of ideas to support the delivery of HIVST. The NGT limited bias/ domination of one cadre/participant over others through ranking. Despite the above-mentioned strengths, the study had some limitations. Firstly, since both the users and stakeholders were in the same room it may have resulted in users being influenced by the presence of providers and not express their views freely, or viceversa. The NGT workshop did not include determination of the acceptability of the proposed HIVST approaches in the current context to help prepare for implementation. A follow-up study to determine the acceptability of the suggested delivery strategies is recommended before implementation.

\section{Conclusion}

This study provides a unique opportunity for development of strategies to help improve uptake of HIV testing services as part of urban development trends to promotes health in South Africa. Improving the uptake of HIV testing by developing tailored HIVST delivery strategies for underserved population such as urban men is key to reducing new HIV infections.

\section{Declarations}

Funding: Canadian Institutes of Health Research HIV Clinical Trials Network (CTN) International Fellowship Program

\section{Ethical approval and consent to participate}

We commenced data collection after obtaining the full ethical clearance from the University of KwaZuluNatal's Biomedical Research Ethics Committee (Reference No. BREC/0000036/2019), the KwaZulu-Natal Provincial Department of Health (Reference No. HRKM055/18, KZ_201911_013). All study participants signed informed consent before participating in the study.

\section{Consent for publication}

Not applicable

\section{Availability of data and materials}

The raw data analysed in this study is available upon reasonable written request submitted to the corresponding author. 


\section{Competing Interests}

None declared.

\section{Funding Statement}

This study is funded in part by the Canadian Institutes of Health Research HIV Clinical Trials Network (CTN) International Fellowship Program

Author contributions: TPM-T conceptualised the study under the supervision of RL and LT; TD assisted with refining the methodology. TPMT and RL conducted data collection; $L T, R L$ and TD critically reviewed the draft of the manuscript. All authors approved the final draft of the manuscript.

\section{Acknowledgements}

A special thanks to all the stakeholders to participated in this workshop. A special thanks to Portia Vezi who assisted in organising this workshop. We would also like to thank Delarise Mulqueeny and Rowan Thompson for their assistance with recruiting some of the workshop participants. We would also like to acknowledge the following organisations for providing stakeholders who participated in this project: eThekwini District Health; KwaZulu-Natal Department of Health; PSI; AHF; CD4WARD; University of KwaZulu-Natal; Umbury Institute, Durban Campus.

\section{References}

1. UNAIDS. Blind spot - Reaching out to men and boys addressing a blind spot in the response to HIV. 2017. [publisher or website?].

2. Pascoe L, Peacock D, Stemple LJIJoMS, Health C. Reaching men: addressing the blind spot in the HIV response. 2018;1(SP1):e57-e70.

3. El-Sadr WM, Harripersaud K, Rabkin M. Reaching global HIV/AIDS goals: What got us here, won't get us there. PLoS Med. 2017;14(11):e1002421.

4. Marcus Low and Sean MacDonell. Graphs that tell the story of HIV in South Africa's provinces. Available at: https://www.spotlightnsp.co.za/2019/08/05/graphs-that-tell-the-story-of-hiv-in-southafricas-provinces/ Accessed on: 23 June 2020. 2019.

5. Launch of the South African National HIV Prevalence, Incidence, Behaviour and. Survey C. 2017. Available at: http://www.hsrc.ac.za/en/media-briefs/hiv-aids-stis-and-tb/sabssm-launch-2018v2. Accesed on: 12 December 2018 [press release]. 2018.

6. Candy Day AG. Health and Related Indicators. HST SAHR. 2017.[Pubisher or website?].

7. Ochieng-Ooko V, Ochieng D, Sidle JE, Holdsworth M, Wools-Kaloustian K, Siika AM, et al. Influence of gender on loss to follow-up in a large HIV treatment programme in western Kenya. Bull World Health Organ. 2010;88:681-8. 
8. Kranzer K, Lewis JJ, Ford N, Zeinecker J, Orrell C, Lawn SD, et al Treatment interruption in a primary care antiretroviral therapy programme in South Africa: cohort analysis of trends and risk factors. Journal of acquired immune deficiency syndromes (1999). 2010;55(3):e17.

9. Cornell M, Schomaker M, Garone DB, Giddy J, Hoffmann CJ, Lessells R, et al. Gender differences in survival among adult patients starting antiretroviral therapy in South Africa: a multicentre cohort study. PLoS Med. 2012;9(9):e1001304.

10. PEPFAR. South Africa Country Operational Plan. 2017. https://www.pepfar.gov/documents/organization/272022.pdf. Accessed on 3 January 2019. 2017.

11. Hensen B, Taoka S, Lewis JJ, Weiss HA, Hargreaves J. Systematic review of strategies to increase men's HIV-testing in sub-Saharan Africa. AIDS. 2014;28(14):2133-45.

12. WHO. Guidelines on HIV self-testing and partner notification: Supplement to consolidated guidelines on HIV testing services. 2016.[website?].

13. Glasgow RE, Lichtenstein E, Marcus AC. Why don't we see more translation of health promotion research to practice? Rethinking the efficacy-to-effectiveness transition. Am J Public Health. 2003;93(8):1261-7.

14. Estem KS, Catania J, Klausner JD. HIV Self-Testing: a Review of Current Implementation and Fidelity. Current HIV/AIDS Reports. 2016;13(2):107-15.

15. Harichund C, Moshabela M. Acceptability of HIV Self-Testing in Sub-Saharan Africa: Scoping Study. AIDS and Behavior. 2017.

16. Colvin CJ. Strategies for engaging men in HIV services. The Lancet HIV. .[Volume: pages?]; 2019.

17. Zhang XQ. The trends, promises and challenges of urbanisation in the world. Habitat international. 2016;54:241-52.

18. Blekking J, Waldman K, Tuholske C, Evans T. Formal/informal employment and urban food security in Sub-Saharan Africa. Appl Geogr. 2020;114:102-31.

19. Venter F, Majam M, Jankelowitz L, Adams S, Moorhouse M, Carmona S, et al. South African HIV selftesting policy and guidance considerations. Southern African journal of HIV medicine. 2017;18(1).

20. Delbecq AL, Van de Ven AH. A group process model for problem identification and program planning. The Journal of Applied Behavioral Science. 1971;7(4):466-92.

21. Mokgetse M, Ramukumba MM. Female condom acceptability and use amongst young women in Botswana. Curationis. 2018;41(1):1-6.

22. Thankian K, Mwaba S, Jere-Folotiya J, Hapunda G, Menon A. Determinants of condom use among currently married men in Zambia. AFRREV IJAH: An International Journal of Arts Humanities. 2017;6(3):65-79.

23. Kabeta T, Belina M, Nigatu M. Hiv voluntary counseling and testing uptake and associated factors among sexually active men in Ethiopia: analysis of the 2016 Ethiopian demographic and health survey data. HIV/AIDS (Auckland, NZ). 2020;12:351. 
24. Chan PS-f, Chidgey A, Lau J, Ip M, Lau JT, Wang Z. Effectiveness of A Novel HIV Self-Testing Service with Online Real-Time Counseling Support (HIVST-online) in Increasing HIV Testing Rate and Repeated HIV Testing among Men Who Have Sex with Men in Hong Kong: Results of a Pilot Implementation Project. International Journal of Environmental Research Public Health. 2021;18(2):729.

25. Hatzold K, Gudukeya S, Mutseta MN, Chilongosi R, Nalubamba M, Nkhoma C, et al. HIV self-testing: breaking the barriers to uptake of testing among men and adolescents in sub-Saharan Africa, experiences from STAR demonstration projects in Malawi, Zambia and Zimbabwe. J Int AIDS Soc. 2019;22:e25244.

26. GSMA. The Mobile EconomySub-Saharan Africa. Accessed on 2. May 2021. Available via: https://www.gsma.com/mobileeconomy/sub-saharan-africa/ 2021 [.

27. Cao B, Gupta S, Wang J, Hightow-Weidman LB, Muessig KE, Tang W, et al. Social media interventions to promote HIV testing, linkage, adherence, and retention: systematic review and meta-analysis. $\mathrm{J}$ Med Internet Res. 2017;19(11):e394.

28. Makusha T, Knight L, Taegtmeyer M, Tulloch O, Davids A, Lim J, et al. HIV self-testing could "revolutionize testing in South Africa, but it has got to be done properly": perceptions of key stakeholders. PloS one. 2015;10(3):e0122783.

29. Gumede SD, Sibiya MN. Health care users' knowledge, attitudes and perceptions of HIV self-testing at selected gateway clinics at eThekwini district, KwaZulu-Natal province, South Africa. SAHARA-J: Journal of Social Aspects of HIV/AIDS. 2018;15(1):103-9.

30. Mathenjwa T, Kim H-Y, Zuma T, Shahmanesh M, Seeley J, Matthews P, et al. Home-based intervention to test and start (HITS) protocol: a cluster-randomized controlled trial to reduce HIV-related mortality in men and HIV incidence in women through increased coverage of HIV treatment. BMC Public Health. 2019;19(1):1-10.

\section{Figures}




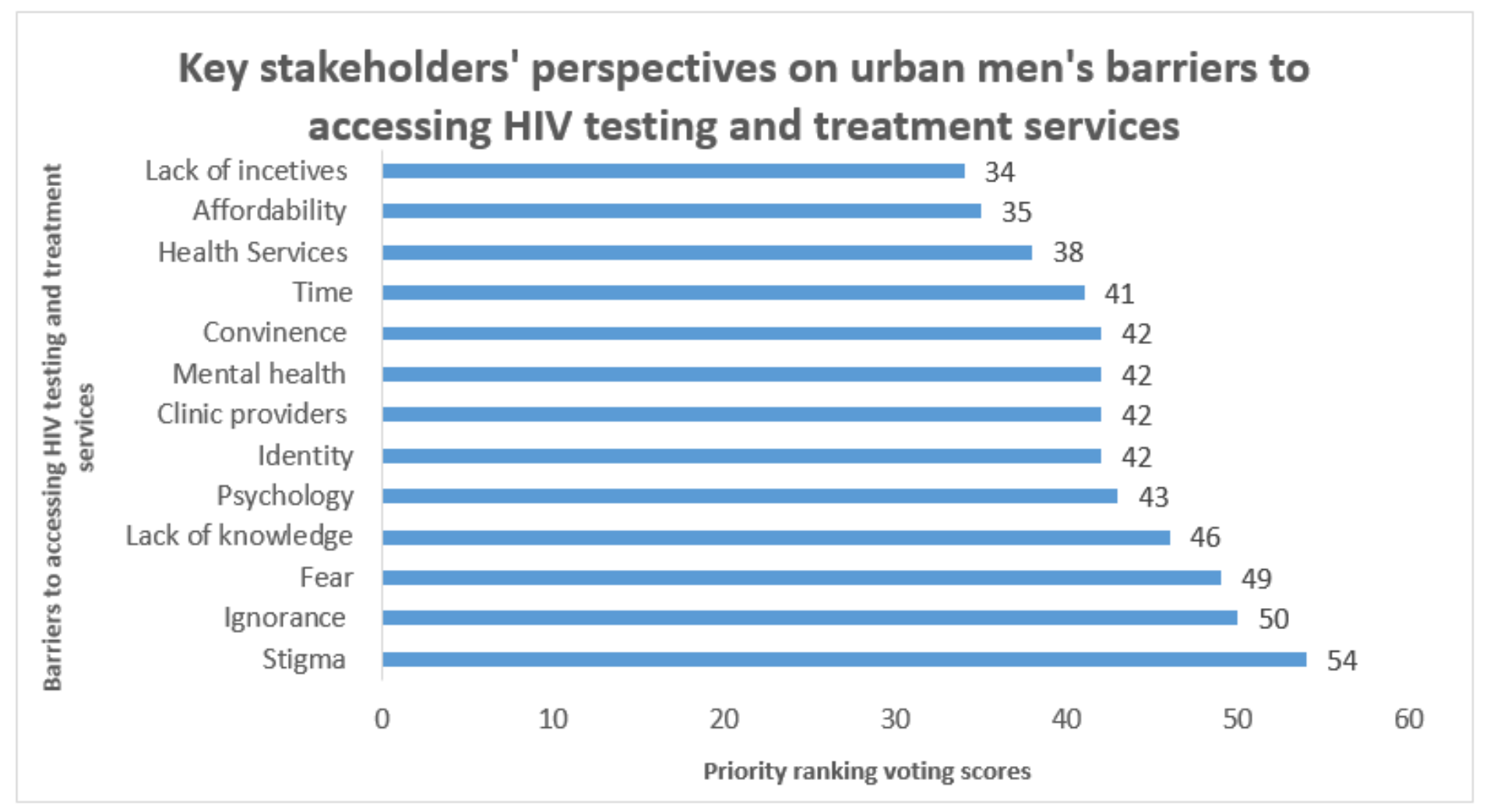

Figure 1

Key stakeholders' voting scores for urban men's barriers to accessing HIV self-testing.

\section{Supplementary Files}

This is a list of supplementary files associated with this preprint. Click to download.

- CONSORT2010checklist.docx 